\title{
On the numerical simulation of fatigue driven delamination with interface elements
}

\author{
J.J. Muñoz, U. Galvanetto *, P. Robinson \\ Department of Aeronautics, Technology and Medicine, Imperial College of Science, Prince Consort Road, London SW7 2AZ, UK
}

\begin{abstract}
This paper investigates robustness and efficiency of a method for the numerical simulation of fatigue driven delamination growth in laminated composites. The method is based on the fatigue degradation of a bilinear interface element and the paper studies the depen dence of the predicted behaviour on the interface element size, $\Delta l$, and the number of cycles per increment, $\Delta N$. It is concluded that the value of $\Delta N$ must be small enough to ensure that at least two increments are required to advance the crack by $\Delta l$. It is also shown that $\Delta l$ must be sufficiently small so that at least two interface elements lie in the cohesive zone ahead of the crack tip. The paper also proposes extensions of the model to include a non zero minimum cyclic load value and an improved algorithm for mixed mode fatigue driven delamination growth.
\end{abstract}

Keywords: Delamination; Fatigue; Interface finite element; Composites

\section{Introduction}

Laminated fibre reinforced plastics are susceptible to delamination, i.e., the separation of the layers. Delamination can be caused by impacts, edge effects, defects or other sources of significant interlaminar stresses. Delamination growth can also be caused by fatigue, which is the deterioration of the interface caused by a large number of load cycles.

Experimental and modelling research has extensively examined the applicability of fracture mechanics to the problem of crack growth in composite materials subject to monotonic loading. Experimental work has involved measurement of the fracture mechanics parameters that characterise the resistance to delamination growth and their sensitivity to environmental conditions such as temperature and moisture. The modelling approaches for the prediction of delamination growth in laminated compos-

\footnotetext{
* Corresponding author. Tel.: +44 (0) 207594 5150; fax: +44 (0) 207584 8120.

E mail address: u.galvanetto@imperial.ac.uk (U. Galvanetto).
}

ites were initially based on a direct application of fracture mechanics and were performed using finite element models of the composite structure. This involved calculating the distribution of the energy release rate, $G$, including the modal components, along the crack front of an initial crack. Various techniques are available for determining $G$ such as the $J$-integral [1] and the virtual crack extension approach [2], but probably the most common approach is the virtual crack closure technique [3]. Once evaluated, the $G$ distribution is tested in a criterion involving the critical energy release rates, measured experimentally, to identify if the crack front propagates under the current loading. The crack front could then be advanced within the finite element model either by moving the finite element mesh or by disconnecting nodes between elements.

More recently, an indirect application of fracture mechanics has been applied to the simulation of delamination in laminated composites [4 9]. This involves the use of an interface element lying along a potential delamination plane. The interface element is assigned a suitable failure characteristic so that it effectively yields and finally 


\section{List of symbols}

\begin{tabular}{|c|c|c|c|}
\hline$a$ & crack length & $x, y$ & coordinate directions in the global axes \\
\hline$C$ & one of the parameters of the fatigue law & $\alpha$ & a power term controlling the mixed mode failure \\
\hline$D_{\mathrm{s}}$ & damage due to static increment of relative dis- & & process \\
\hline & placement & $\beta$ & one of the parameters of the fatigue law \\
\hline$D_{\mathrm{f}}$ & damage due to fatigue & $\delta_{1}$ & relative displacement component in mode I \\
\hline$G$ & energy release rate & $\delta_{2}$ & relative displacement component in mode II \\
\hline$G_{\mathrm{c}}$ & critical energy release rate & $\delta_{0 i}$ & elastic limit of the relative displacement compo- \\
\hline$G_{\min }$, & $G_{\max }$ minimum and maximum $G$ in a cycle & & nent in mode $i$ \\
\hline$N$ & number of loading cycles & $\delta_{\mathrm{c} i}$ & failure limit of the relative displacement compo- \\
\hline$t_{1}$ & stress component associated with mode I & & nent in mode $i$ \\
\hline$t_{2}$ & stress component associated with mode II & $\Delta l$ & interface element length \\
\hline$t_{0 i}$ & elastic limit of the stress associated with mode $i$ & $\Delta N$ & number of cycles per increment \\
\hline$u, v$ & $\begin{array}{l}\text { relative displacement components in the global } \\
\text { axes }\end{array}$ & $\begin{array}{l}\lambda \\
\xi, \eta\end{array}$ & $\begin{array}{l}\text { one of the parameters of the fatigue law } \\
\text { coordinate directions in the local axes }\end{array}$ \\
\hline
\end{tabular}

separates when energy equivalent to the critical energy release rate has been absorbed. The interface element has a number of advantages over the direct use of fracture mechanics. One key advantage is associated with representing the propagation of a planar crack; the interface element essentially fails where appropriate, whereas for the direct use of fracture mechanics an intricate mesh moving algorithm is required to deal with the full complexity of realistic structural configurations. (Disconnecting nodes to advance the crack front is only appropriate if the crack growth problem can be reduced to that of a line crack in a $2 \mathrm{D}$ mesh.) A further advantage of the interface element approach is that it can potentially model the initiation of a crack where none existed previously. This does, however, mean that the parameters associated with the interface element failure law need to be determined more rigorously than for the crack propagation analyses $[10,11]$.

The propagation of fatigue-driven crack growth in composite materials has also received considerable attention. Again experimental work has been performed to measure crack growth rates as a function of cyclic loading parameters and environmental conditioning. A basic approach to representing the crack growth rate is to apply the Paris law: $\mathrm{d} a / \mathrm{d} N \quad A \Delta G^{\mathrm{m}}$ where $a$ is the crack length, $N$ the number of cycles, $\Delta G$ is the range of the strain energy release rate applied cyclically and $A$ and $\mathrm{m}$ are material constants [12]. However, the trend of a typical curve of $\log (\mathrm{d} a / \mathrm{d} N)$ versus $\log (G)$ is not linear, as implied by the Paris law, but has a sigmoid shape; the lower limit relates to no growth below a certain $G$ (the threshold value) and an upper limit is associated with rapidly increasing crack growth rate which occurs when the maximum value of the cyclic applied $G_{\max }$ approaches the critical value, $G_{\mathrm{c}}$. A modified Paris law has therefore been defined which describes the full $\mathrm{d} a / \mathrm{d} N$ versus $G_{\max }$ curve, i.e., including the threshold and accelerating crack growth regions [13]. The modified Paris law is given by

$\left.\frac{\mathrm{d} a}{\mathrm{~d} N}=B G_{\max }^{\mathrm{m}} \frac{1-\left(G_{\mathrm{th}} / G_{\max }\right)^{n 1}}{1-\left(G_{\max } / G_{\mathrm{c}}\right)^{n 2}}\right)$,

where $G_{\text {th }}$ is the fatigue threshold, that is, the value of the energy release rate below which no crack propagation is observed, and $G_{\mathrm{c}}$ the critical energy release rate, that is, the value of the energy release rate that would generate crack propagation independent of the cyclic nature of the loading. The constants $\mathrm{m}, n 1, n 2$ and $B$ can be obtained by fitting this expression to the experimental data. (It should be noted that the values of the constants will be a function of the ratio $G_{\max } / G_{\min }$ and that the above expression was proposed initially for mode I driven growth; a general expression will need to address mixed-mode fatigue.) Crack growth rate laws like the Paris law and the modified Paris law can be applied in conjunction with FE models incorporating evaluation of the energy release rate around the crack front, determined as described earlier. The crack growth rate can then be evaluated according to the rate law and the crack front advanced an appropriately small amount corresponding to an increment in $N$. (For a crack growth problem that can be reduced to that of a line crack in a 2D mesh, the variation of $G$ with crack length $a$ can be determined from the FE model and then the crack growth rate law can be re-arranged and integrated to give the crack length as a function of $N$.)

An alternative approach for the simulation of fatigue driven delamination growth is to incorporate fatigue degradation into the interface element technique for modelling crack propagation. The use of the interface element technique for prediction of fatigue-driven crack propagation has the advantages mentioned earlier for monotonic loading: the avoidance of the re-meshing problems and the potential to predict the fatigue initiation of a crack where no crack existed initially. The interface element approach should also be able to deal with realistic situations where, for example, crack growth may initially occur principally due to the 
maximum applied $G$ exceeding the monotonic loading crack growth criterion and in later stages the growth may be dominated by the cyclic nature of the loading.

The present paper investigates a particular numerical method developed to simulate fatigue driven delamination growth in laminated composites. The approach is based on the use of interface elements, the constitutive law of which has been modified by introducing a fatigue degradation algorithm. The goal of the numerical method is limited to reproduce the well-known linear portion of the Paris plot, which describes the rate of growth of the crack length per cycle as a function of the applied strain energy release rate. The method, based on the cohesive-zone model pioneered by Dugdale and Barenblatt [14,15], is described in Ref. [16]: a fatigue law initially proposed for continuum mechanics in Ref. [17] was introduced in the interface element proposed by Crisfield and co-workers in Refs. [8,9] to simulate delamination due to fatigue. In Ref. [16] the number of cycles $N$ of the external load is considered as a pseudo-time variable and the external load varies during a cycle between a fixed maximum value and zero. The present paper focuses on numerical issues associated with the implementation and a possible enhancement of the formulation. The development of the interface element to incorporate fatigue damage has been implemented in the finite element code LUSAS [18]. The algorithm presented in this paper has been applied only to 2D problems where a 1D crack line can be identified. Planar interface elements are available, for example in Ref. [18], and, in principle, their constitutive law can be equipped with a similar fatigue degradation algorithm. However, it is clear that in a 3D case the crack would be, at least, bi-dimensional and additional problems arising in modelling and convergence should be addressed. Similar issues are discussed in the recent papers published on 3D fatigue crack growth, such as [19].

The numerical procedure proposed in Ref. [16] is briefly summarised in Section 2, where also a comparison between the experimental and numerical data is presented, and then, in Section 3, the results of investigations into the following aspects are presented:

(a) Dependence of the numerical solution on $\Delta N$, the number of cycles per load increment (Section 3.1).

(b) Mesh dependence (Section 3.2).

(c) The introduction of a non-zero minimum cyclic load (Section 3.3).

In Section 4, an alternative formulation for the mixed mode delamination is presented and finally the conclusions of the work are presented in Section 5.

\section{Interface elements and fatigue law}

In this section, a concise description of the interface element is given and, in particular, of the fatigue part of the constitutive law. The reader is referred to Refs. $[16,17]$ for a more complete description of the fatigue law.

\subsection{Preliminary definitions}

The present section is largely based on Ref. [9]. The geometry of a 4-noded interface element, which is a straight line forming an angle $\alpha$ with the $x$-axis is illustrated in Fig. 1a. The corresponding reference element, which is related to the real one via the standard isoparametric mapping is shown in Fig. 1b. Nodes 1 and 2 represent one side of the interface, nodes 3 and 4 represent the other. Each node has two degrees of freedom, $u$ and $v$, with reference to the global axes $x$ and $y$. In each element, a local reference system is defined and the relative displacement between each pair of the corresponding upper and lower nodes is the vector addition of an opening (mode I) component and a sliding (mode II) component. At a generic point of the interface, the relative displacement will be denoted by $\boldsymbol{\delta}^{\mathrm{T}} \quad\left(\begin{array}{lll}\delta_{1} & \delta_{2}\end{array}\right)$ while $\boldsymbol{t}^{\mathrm{T}} \quad\left(\begin{array}{ll}t_{1} & t_{2}\end{array}\right)$ will indicate the corresponding interface traction, in which subscripts 1 and 2 indicate the opening and sliding components, respectively, i.e., vectors $\boldsymbol{\delta}$ and $\boldsymbol{t}$ are expressed in the local reference system.

The relationship between the vector of stresses $\boldsymbol{t}$ and the relative displacements $\boldsymbol{\delta}$ can be written as

$$
\boldsymbol{t}=(\mathbf{I}-\mathbf{D}) \mathbf{K} \boldsymbol{\delta}
$$

with $\mathbf{K}$, the initial stiffness, and $\mathbf{D}$, the matrix of damage parameters, given by
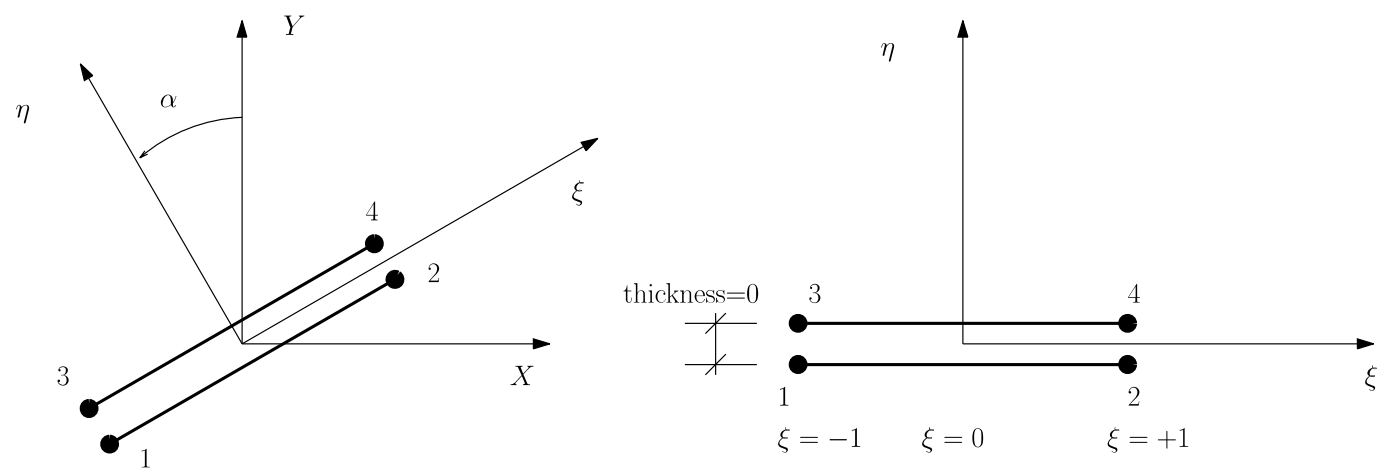

Fig. 1. Interface element: (a) global system and (b) local system. 


$$
\begin{aligned}
& \mathbf{K}=\left[\begin{array}{cc}
K_{1} & 0 \\
0 & K_{2}
\end{array}\right]=\left[\begin{array}{cc}
\frac{t_{01}}{\delta_{01}} & 0 \\
0 & \frac{t_{02}}{\delta_{02}}
\end{array}\right], \\
& \mathbf{D}=\left\{\begin{array}{cl}
\frac{\gamma^{*}}{\gamma^{*}+1}\left[\begin{array}{cc}
F_{1} & 0 \\
0 & F_{2}
\end{array}\right] & \text { if } \gamma^{*}>0 \\
0 & \text { if } \gamma^{*} \leqslant 0
\end{array}\right.
\end{aligned}
$$

in which

$$
\begin{aligned}
& F_{i}=\frac{\delta_{\mathrm{c} i}}{\delta_{\mathrm{c} i}-\delta_{0 i}}, \\
& \gamma^{*}=\max _{\tau}\left(\gamma_{\tau}\right), \\
& \gamma=\left[\left(\frac{\delta_{1}}{\delta_{01}}\right)^{\alpha}+\left(\frac{\delta_{2}}{\delta_{02}}\right)^{\alpha}\right]^{\frac{1}{\alpha}}-1 .
\end{aligned}
$$

The relative displacements $\delta_{0 i}$ and $\delta_{\mathrm{c} i}$ are the elastic limit and the failure limit of the relative displacement, respectively, and for $\delta_{i}>\delta_{\mathrm{c} i}$ the two surfaces of the interface element are considered completely disconnected. The variable $\tau$ is the pseudo-time denoting the load history. The definition of $\gamma$ in Eq. (3b) provides also the single mode delamination by assuming that one of the two relative displacements, either $\delta_{1}$ or $\delta_{2}$, is zero (see Fig. 2). Moreover, it provides a smooth transition between single mode and mixed mode delamination in case that a component of the relative displacements, initially equal to zero, becomes different from zero. In general it would be possible to assume $F_{1} \neq F_{2}$, and therefore $D_{1} \neq D_{2}$. Such an assumption involves the undesirable consequence that one component of damage could reach the failure value of 1 before the other and that would correspond to the unrealistic case of an interface completely failed in mode I but with still a certain resistance in mode II. The mathematical relationships in the next sections are written for the general case $F_{1} \neq F_{2}$, but the numerical examples shown in Section 3 have been obtained with the condition $F_{1} \quad F_{2}$.

In the computations carried out to simulate fatigue behaviour, no unloading behaviour needs to be defined. Ref. [9] provides a complete description of the constitutive law for the interface element under static load.

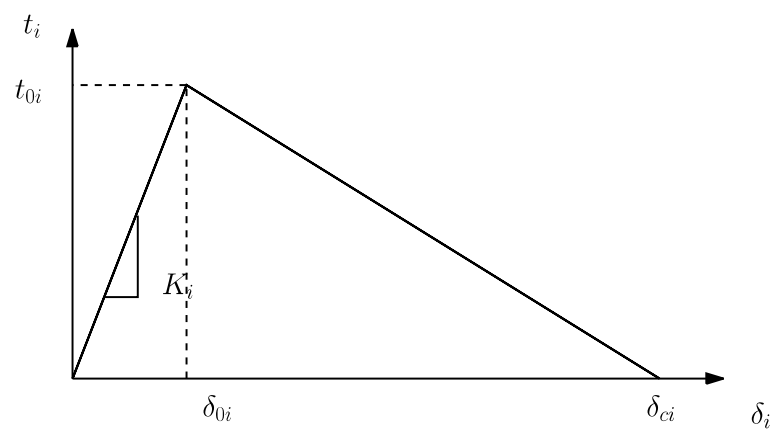

Fig. 2. Bilinear and convex stress displacement relationship.

\subsection{Damage evolution due to fatigue}

Some assumptions were made in Ref. [16] in order to simplify the calculation process. The cyclic load that has to be simulated (in terms of applied displacement or forces) was assumed sinusoidal, oscillating between zero and a maximum value. Since the aim of this investigation is to simulate high-cycle fatigue, it would be extremely demanding to simulate the effective relative displacement history for each pair of nodes of the interface elements. For constant amplitude loading, the load numerically applied to the structure will therefore be taken as constant and equal to the maximum value of the actual cyclic load and consequently the relative displacement calculated at the interface will be considered as the envelope of its cyclic variation with time (see Fig. 3). The relative displacement calculated at the interface must be interpreted as the momentary amplitude rather than the actual value of the relative displacement, as clearly explained in Ref. [17]. Furthermore, since an elastic/damage model is used, no permanent deformation develops and minimum local magnitudes of strains, stresses and relative displacements at the interface are zero. The number of cycles will be considered as a real-valued variable and represents the pseudo-time of the structure, the word 'pseudo' being used to indicate that a rate-independent model is assumed and that inertia effects are neglected.

Following the work described in Refs. [16,20], the rate of total damage $\dot{D}_{i}$, is split in static damage $\dot{D}_{i, s}$, and fatigue damage $\dot{D}_{i, \mathrm{f}}$, i.e.,

$\dot{D}_{i}=\dot{D}_{i, \mathrm{~s}}+\dot{D}_{i, \mathrm{f}}$.

For single mode delamination, the rate of the static damage component can be written as follows:

$\dot{D}_{i, \mathrm{~s}}=\left(\frac{\delta_{0 i} \delta_{\mathrm{c} i}}{\delta_{\mathrm{c} i}-\delta_{0 i}}\right) \frac{\dot{\delta}_{i}}{\delta_{i}^{2}} \quad$ with $\dot{\delta}_{i} \geqslant 0$

Similarly for single mode delamination the rate of the fatigue damage component can be written by adapting Peerlings' law [17] as follows:

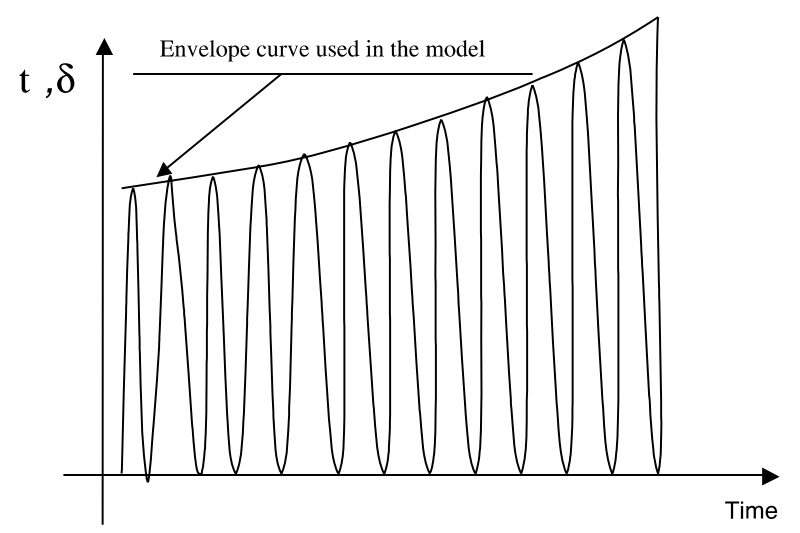

Fig. 3. Envelope curve of the traction and relative displacement in an interface element. 
$\dot{D}_{i, \mathrm{f}}=C \mathrm{e}^{\lambda D_{i}}\left(\frac{\delta_{i}}{\delta_{\mathrm{c} i}}\right)^{\beta} \frac{\dot{\delta}_{i}}{\delta_{\mathrm{c} i}}$,

where $C, \lambda$ and $\beta$ are parameters of the model which have to be determined by comparison with experimental data. This law allows the damage to grow with the number of cycles even if the initial damage of the interface is zero. Thus, using Peerlings's law, a crack can grow and propagate even in an initially undamaged interface.

Similarly, the evolution law for the damage in coupled mode is written as:

$\dot{D}_{i, s}=F_{i} \frac{\dot{\gamma}}{(1+\gamma)^{2}}$,

$\dot{D}_{i, \mathrm{f}}=C \mathrm{e}^{\lambda D_{i}}\left(\frac{1+\gamma}{1+\gamma_{\mathrm{c}}}\right)^{\beta} \frac{(1 \dot{+} \gamma)}{1+\gamma_{\mathrm{c}}}$

with

$\gamma_{\mathrm{c}}= \begin{cases}{\left[\left(\frac{\delta_{\mathrm{c} 1}}{\delta_{01}}\right)^{\alpha}+\left(\frac{\delta_{\mathrm{c} 2}}{\delta_{02}}\right)^{\alpha}\right]^{\frac{1}{\alpha}}-1} & \text { if } \delta_{1} \neq 0 \text { and } \delta_{2} \neq 0, \\ \frac{\delta_{\mathrm{cl}}}{\delta_{01}}-1 & \text { if } \delta_{1} \neq 0 \text { and } \delta_{2}=0, \\ \frac{\delta_{\mathrm{c} 2}}{\delta_{02}}-1 & \text { if } \delta_{1}=0 \text { and } \delta_{2} \neq 0 .\end{cases}$

Note that the definition of $\gamma_{c}$ comprises the single mode cases, but the transition from single mode to mixed mode, and vice versa, would not be smooth. An alternative, continuous definition of $\gamma_{c}$ is discussed in Section 4.

The integration of the damage rate $\dot{D}_{i}$ in (4) over $\Delta N$ cycles leads to the following expression of the increment of damage $\Delta D_{i} \quad D_{i, N+\Delta N}-D_{i, N}[16]$ :

$\Delta D_{i}=\Delta D_{i, \mathrm{~s}}+\Delta_{i, \mathrm{f}}$,

where
$\Delta D_{i, \mathrm{~s}}=F_{i}\left(\frac{1}{1+\gamma_{N}}-\frac{1}{1+\gamma_{N+\Delta N}}\right)$

$\Delta D_{i, \mathrm{f}}=\frac{\Delta N C \mathrm{e}^{\lambda D_{i, \mu}}}{1+\beta}\left(\frac{1+\gamma_{\mu}}{1+\gamma_{\mathrm{c}}}\right)^{\beta+1}$

in which $(\bullet)_{\mu} \quad(1-\mu)(\bullet)_{N}+\mu(\bullet)_{N+\Delta N}$. The increment of damage is computed by solving iteratively the following equation:

$H_{i} \equiv \Delta D_{i}-\Delta D_{i, \mathrm{~s}}-\Delta D_{i, \mathrm{f}}=0$,

where $\Delta D_{i, \mathrm{~s}}$ and $\Delta D_{i, \mathrm{f}}$ are those mentioned in Eq. (10b) and $\mu$ is a parameter which defines the numerical interpolation. In the examples shown in the present paper, $\mu \quad 0.7$. The linearisation of the stress displacement relationship $\boldsymbol{t}(\mathbf{I}-\mathbf{D}) \mathbf{K} \boldsymbol{\delta}$ when considering the total (static and fatigue) damage is given in Appendix A.

In Ref. [16], it has been shown that this formulation can reproduce fatigue crack growth data determined experimentally for interlaminar toughness specimens in mode I, mode II and mixed mode $\left(\left(G_{\mathrm{I}} / G_{\mathrm{II}}\right) \quad 1\right)$. The corresponding experimentally determined and numerically simulated Paris plots are shown in Figs. 4 and 5.

The fatigue law (8) requires the determination of three parameters: $C, \lambda$ and $\beta$. The sensitivity of the fatigue law to their numerical values is an important issue which has been partially addressed in Ref. [16], for the case of interface elements, and in Ref. [17] for the continuum case. In the two references, the parameters $\lambda$ and $\beta$ take positive values of a few units or less. $C$ can vary instead of many orders of magnitude. This issue has to be investigated in greater detail, since a great sensitivity would reduce the potential interest for the numerical method under investigation.
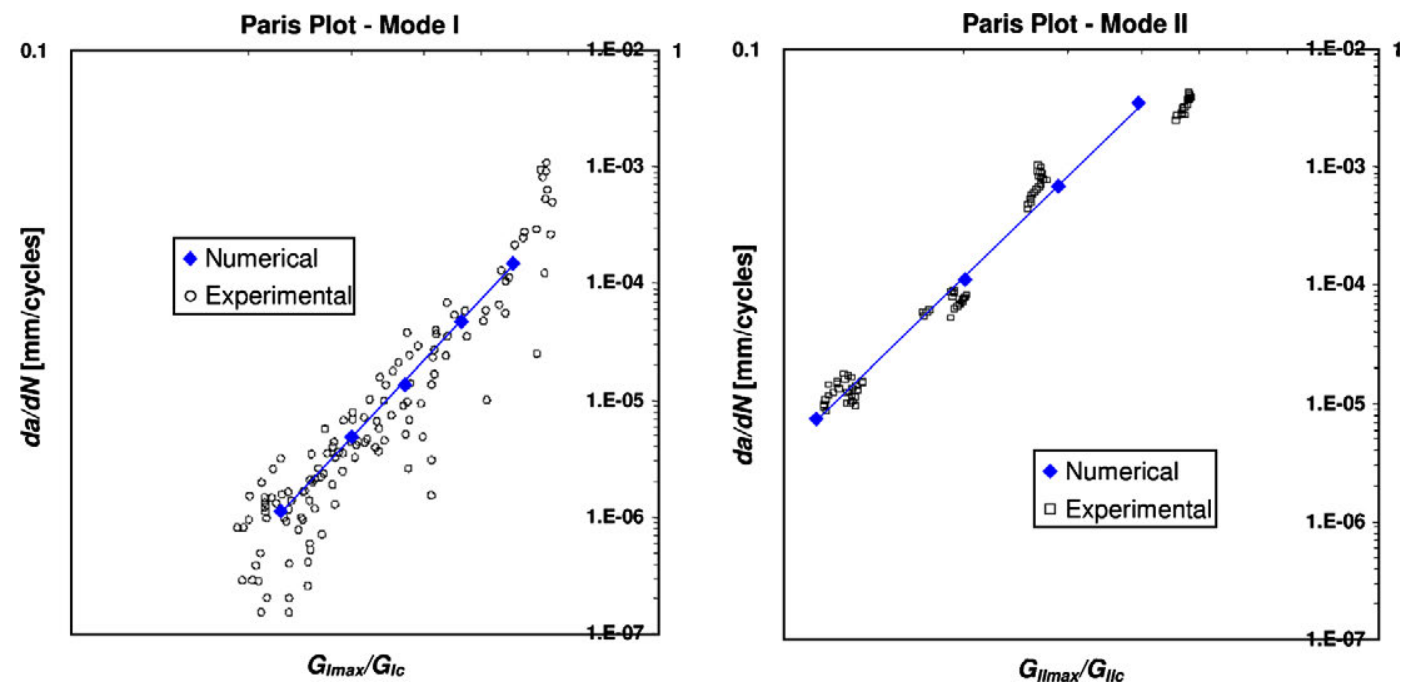

Fig. 4. Paris plot for mode I $\left(C=2 \times 10^{6}, \beta=2\right)$ and mode II $\left(C=3 \times 10^{5}, \beta=2, \lambda=0.5\right)$ with experimental results from [21] and numerical results from [16]. 


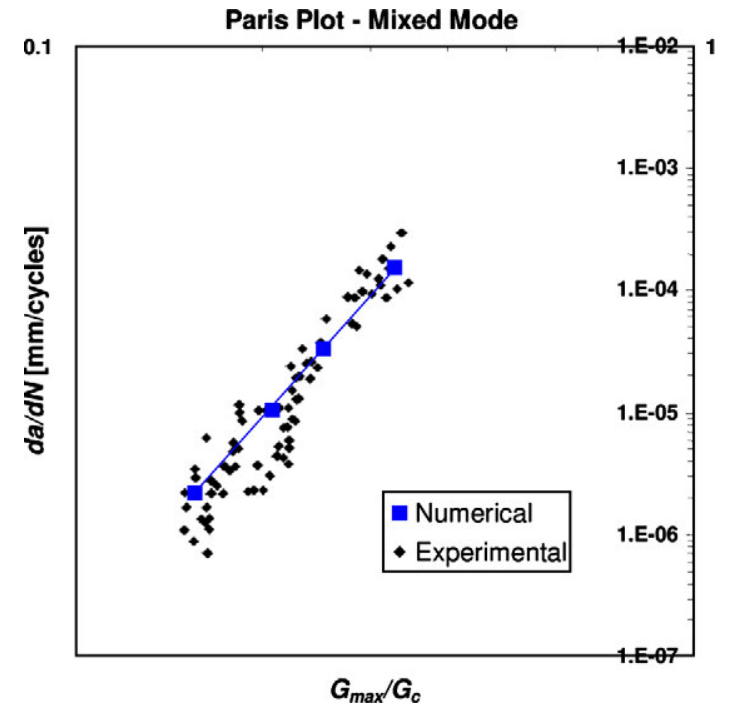

Fig. 5. Paris plot for the mixed mode with experimental results from [21] and numerical results from $[16]\left(C=1 \times 10^{4}, \beta=2.8, \lambda=0.5\right)$.

\section{Numerical issues}

Although the formulation in the previous section is able to successfully reproduce the experimental Paris plots, it is important to understand how the results are affected when varying key parameters such as the pseudo-time-step $\Delta N$ or the mesh size, here denoted by $\Delta l$. Our aim in this section is twofold: to provide valid problem-dependent estimates for $\Delta N$ and $\Delta l$, and, at the same time, investigate the objectivity of the results with respect to these parameters.

\subsection{Choice of $\Delta N$}

Clearly, large values of $\Delta N$ would be very advantageous from the computational cost standpoint. On the other hand, the approximate integration of the damage rate over $\Delta N$ cycles, carried out in [16], is based on the assumption of small increments of damage and therefore the value of $\Delta N$ cannot be too large. Examples will be presented of the effects of using too many cycles per increment. Moreover, it is also important to determine which are the relevant parameters of the structure that constrain the selection of $\Delta N$.

Let us first consider a model of a double cantilever beam (DCB) with a constant applied moment $M$ (dimensions and material parameters are shown in Fig. 6 and a sketch of the mesh is shown in Fig. 7). In order to reduce the number of degrees of freedom of the model, the bulk material will be modelled with beam elements which have a length that will be always smaller or equal to the length of the interface element, $\Delta l$.

Fig. 8 shows the resulting crack evolution for three different values of $\Delta N: 500,1000$ and 2000 cycles/increment. Although different values of $\Delta N$ provide almost identical crack growth rates, for the largest value no convergence was achieved after approximately 46,000 cycles.

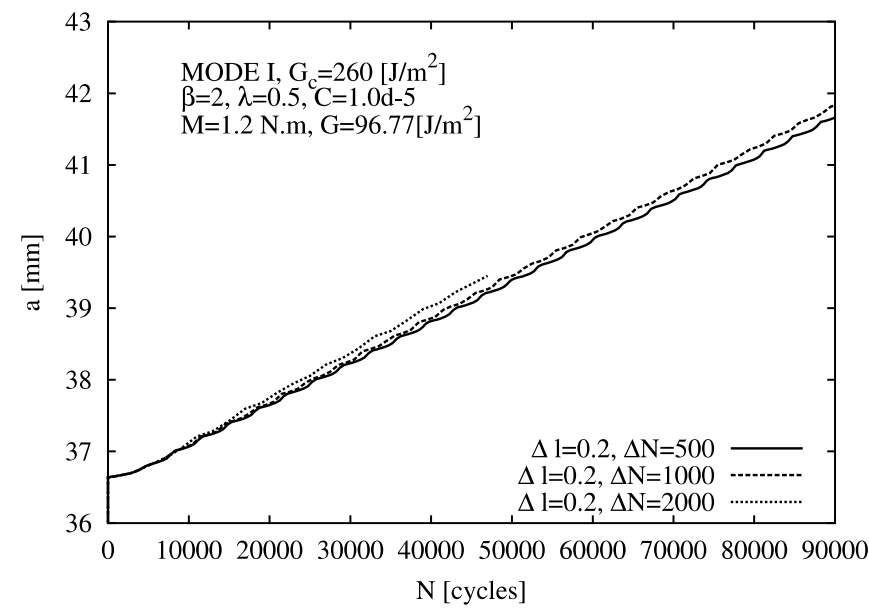

Fig. 8. Crack growths for $\Delta l=0.2 \mathrm{~mm}$ and $\Delta N=500,1000$ and 2000 cycles/increment. $t_{01}=30 \mathrm{~N} / \mathrm{mm}^{2}, \delta_{01}=10^{6} \mathrm{~mm}$.

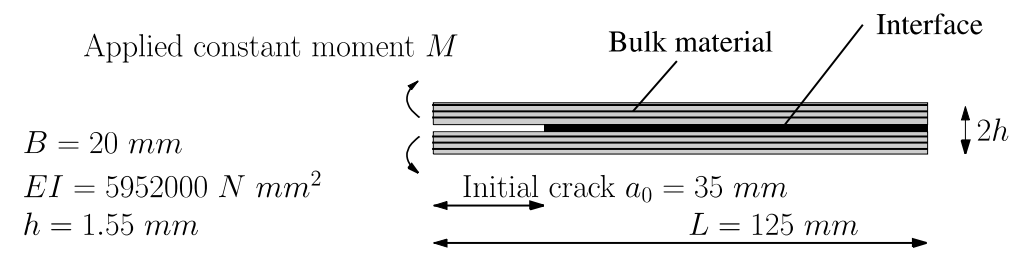

Fig. 6. Scheme and dimensions of the DCB test. $B$ is the width of the beam, the value of EI is for one arm of the DCB.

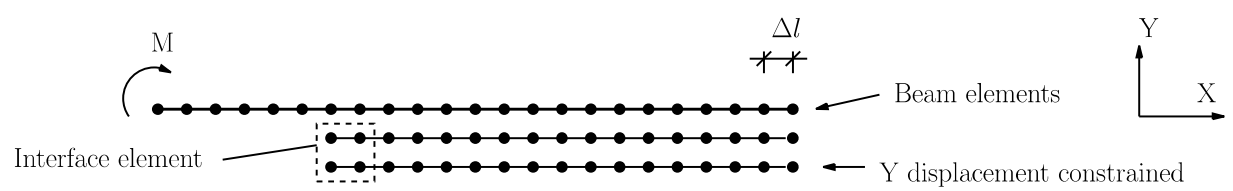

Fig. 7. Scheme of the finite element discretisation. The nodes have been drawn in different layers to ease the visualisation although in the model they all have the same $Y$ co ordinate. 
For other values of $\Delta N$ and $\Delta l$ (but maintaining the same size for the beam elements), similar convergence problems were encountered, but with other critical maximum number of cycles. It is proposed that in order to ensure convergence, the maximum number of cycles per increment should be limited according to the following condition:

The value of $\Delta N$ must be such that at least two increments are required to advance the crack over one element.

This is equivalent to saying that the crack tip must advance less than one half of the element length between successive increments. Unfortunately, this rule can be only applied a posteriori, in the sense that it gives the maximum value once the crack growth rate is known. Nevertheless, it is still a useful rule when the crack growth rate can be roughly estimated.

The same problem shown in Figs. 6 and 7 have been tested with values of $\Delta N$ smaller than 500 , and all of them converged satisfactorily. Of course, though, 'small' values of $\Delta N$ will increase the computational cost, perhaps unnecessarily.

In Figs. 8, 9 and 12, the crack length at zero cycles is not equal to $a_{0} \quad 35 \mathrm{~mm}$ because the crack length is computed in an approximated way. Engineering theory of beams is used to equate the crack length to the length of a perfectly clamped beam which has the same tip deflection as a single arm of the DCB. It is clear that the most important quantity $\mathrm{d} a / \mathrm{d} N$ is not affected by the approximation affecting the computed value of $a$.

\subsection{Mesh dependence}

The conclusions arrived at above seem to suggest that by increasing the element size it would be possible to increase also $\Delta N$, and therefore reduce the number of solution increments and the computational time. However, the pres-

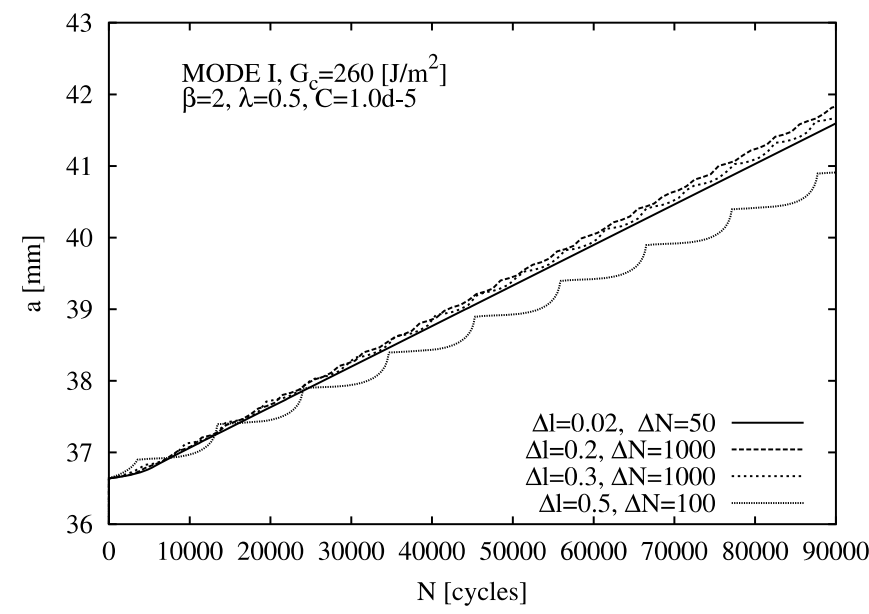

Fig. 9. Crack growths for different mesh sizes. $t_{01}=30 \mathrm{~N} / \mathrm{mm}^{2}$, $\delta_{01}=10^{6} \mathrm{~mm}$. ent section will explain that the maximum value of $\Delta l$ is constrained by the properties of the interface element.

The DCB test described above has been carried out using different values of $\Delta l$. Several crack evolutions for four different elements sizes $\Delta l: 0.5,0.3,0.2$ and $0.02 \mathrm{~mm}$ are shown in Fig. 9. For the smallest element size, the value of $\Delta N$ has been adjusted for convergence reasons, according to the rule established in Section 3.1. For the largest size $\Delta l \quad 0.5 \mathrm{~mm}$, the value of $\Delta N$ has been reduced to 100 due to convergence problems.

It is apparent that all the curves, except that for $\Delta l \quad 0.02$, show a set of undulations, whose wavelength, as stated in [16], corresponds to the element size (the undulations can also be observed in Fig. 8). It is clear that the undulations become more severe as the element size increases. Fig. 9 also shows that the overall slope of the $a$ vs $N$ curve (i.e., the slope of the best fit straight line) departs from a converged value as the undulations become large, i.e., as $\Delta l$ increases.

It is therefore interesting to study the damage profile for the DCB model described in the previous section. The curves in Fig. 10 show the spatial variation of damage $D$ along the interface at a certain crack length, for $\Delta l \quad 0.5$, 0.2 and $0.02 \mathrm{~mm}$, respectively. It is clear that, in all cases, the cohesive zone (i.e., the damaged zone with values of $D$ between 0 and 1 ) is very narrow. For the meshes with element sizes $\Delta l \quad 0.5$ and 0.2 , it is contained within a few elements, and only for $\Delta l \quad 0.02$ can the damage profile, which is spread over 1015 elements, be accurately appreciated.

From these results, it can be inferred that the structural behaviour of the DCB test during the crack growth will be influenced by two main effects: the transition of the damage front from element to element, and the progress of the crack tip within one interface element. The two situations have in fact different influences on the DCB response, and in fact, the larger the element, the stronger will be the influence of the second effect.

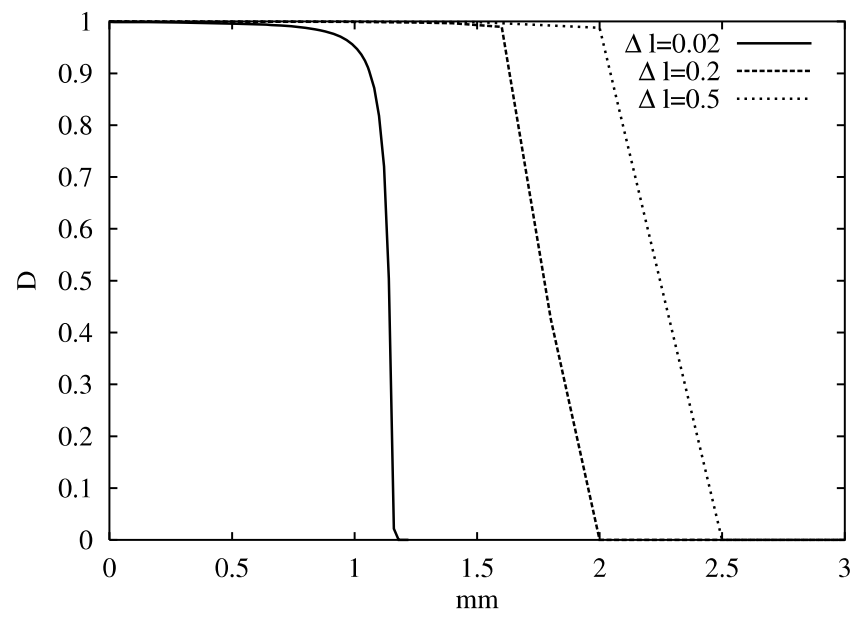

Fig. 10. Damage profile at the crack for $\Delta l=0.02 \mathrm{~mm}, \Delta l=0.2 \mathrm{~mm}$ and $\Delta l=0.5 \mathrm{~mm}$ (and at different number of cycles for clarity). The horizontal axis shows the distance in the crack growth direction in $\mathrm{mm}$. 
Based on the results presented above, to avoid excessive undulations in the $a N$ plot, $\Delta l$ should be sufficiently small so that at least two elements lie in the cohesive zone.

\subsection{Fatigue with $G_{\min } \neq 0$}

The formulation described so far is the adaptation of Peerlings' law in Ref. [17] to the interface element. In doing so, we have considered only the case where the cyclic load (displacement) curve has a constant minimum value of $\left.\begin{array}{lll}t & 0 \text { (or } \delta & 0\end{array}\right)$ (see Fig. 11a). In many practical cases, however, the load (displacement) oscillates between two non-zero values, which is equivalent to considering two non-zero values of $G$, say, $G_{\min }$ and $G_{\max }$ (see Fig. 11b). By defining the factor

$\rho=\frac{G_{\min }}{G_{\max }}$,

which will be assumed constant, we can model an applied cyclic load by replacing the factor $C$ in the damage evolution law in (8) with

$C_{\rho}=C(1-\rho)$.

In Eqs. (12) and (13) a static load is represented by $\rho 1$, which means $G_{\min } G_{\max }$. For simplicity, we have chosen a linear relationship between $C_{\rho}$ and $\rho$. This approach qualitatively reflects the experimentally observed behaviour, but further evaluation and refinement of the approach will be required to accurately simulate delamination growth for cyclic loading with a non-zero minimum value. This is currently an actively debated issue [22]. A set of crack evolutions for different values of $\rho$ is plotted in Fig. 12 for the DCB test shown in Fig. 6. It can be observed that $\frac{\mathrm{d} a}{\mathrm{~d} N}$ decreases as $\rho$ is increased. In particular, if $\rho \quad 1$, the increment of fatigue damage given by Eq. $(10 \mathrm{~b})$ is zero and the corresponding curve shown in Fig. 12 is therefore a horizontal straight line.

\section{Alternative coupled formulation}

The presence of the non-continuous definition of $\gamma_{c}$ in Eq. (9) may lead to convergence problems when the analy-

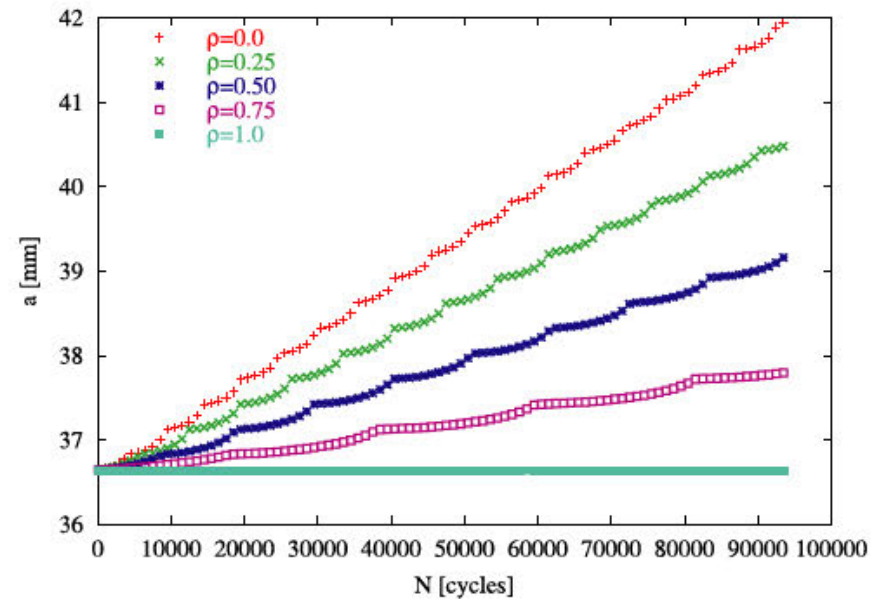

Fig. 12. Crack growths due to fatigue for different ratios $\rho \frac{G_{\min }}{G_{\max }}$.

sis encounters a transition from mixed to single mode or vice versa.

In this section, an alternative approach is proposed which overcomes this problem.

\subsection{Damage evolution due to fatigue}

Instead of using Eq. (8), an alternative adaptation of Peerlings' law results in the following equation:

$\dot{D}_{i, \mathrm{f}}=C \mathrm{e}^{\lambda D_{i}}(1+\gamma)^{\beta}(1 \dot{+} \gamma)$.

In single mode this equation becomes

$\dot{D}_{i, \mathrm{f}}=C \mathrm{e}^{\lambda D_{i}}\left(\frac{\delta_{i}}{\delta_{0 i}}\right)^{\beta} \frac{\dot{\delta}_{i}}{\delta_{0 i}}$.

Note that in comparison to the previous expression in (6), the reference displacement used in the adaptation of Peerlings' law is now $\delta_{0 i}$ rather than $\delta_{\mathrm{c} i}$. The time-integration of $\dot{D}_{i, \mathrm{f}}$ in (14) over $\Delta N$ cycles leads to

$\Delta D_{i, \mathrm{f}}=\Delta N C \mathrm{e}^{\lambda D_{i, \mu}} \frac{\left(1+\gamma_{\mu}\right)^{\beta+1}}{1+\beta}$

and hence, in this case, the equation $H_{i} \quad 0$ (equivalent to (11)) uses the following definition of function $H_{i}$ :
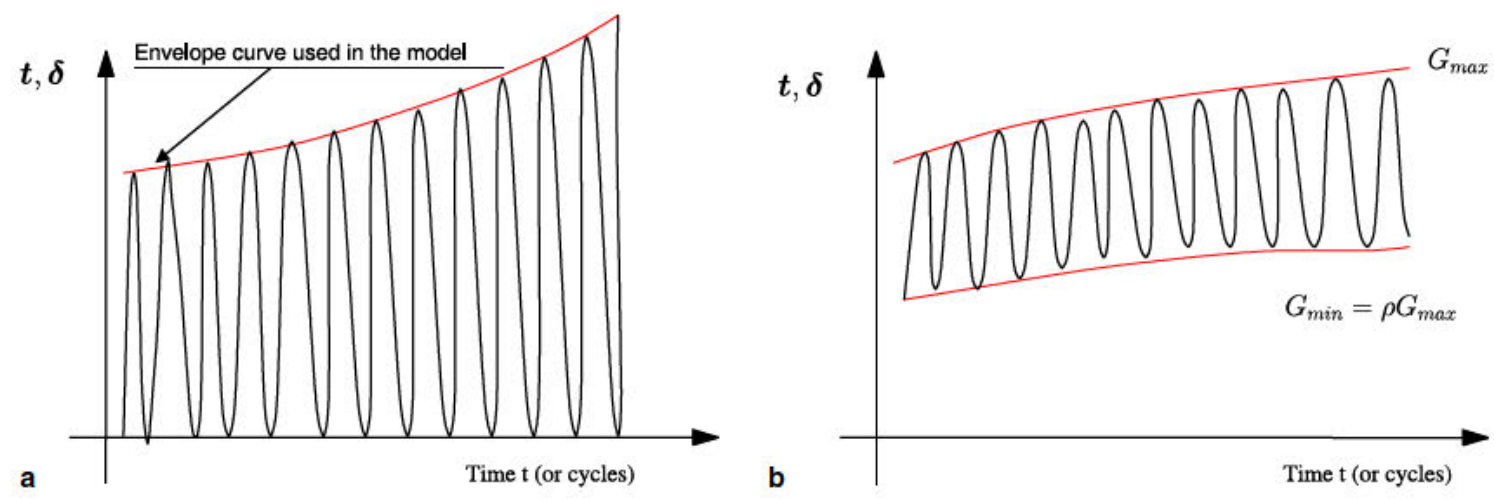

Fig. 11. Scheme of load/displacement cycles with: (a) $G_{\min }=0$ and (b) $G_{\min } \neq 0$. 
Paris Plot - Mode I

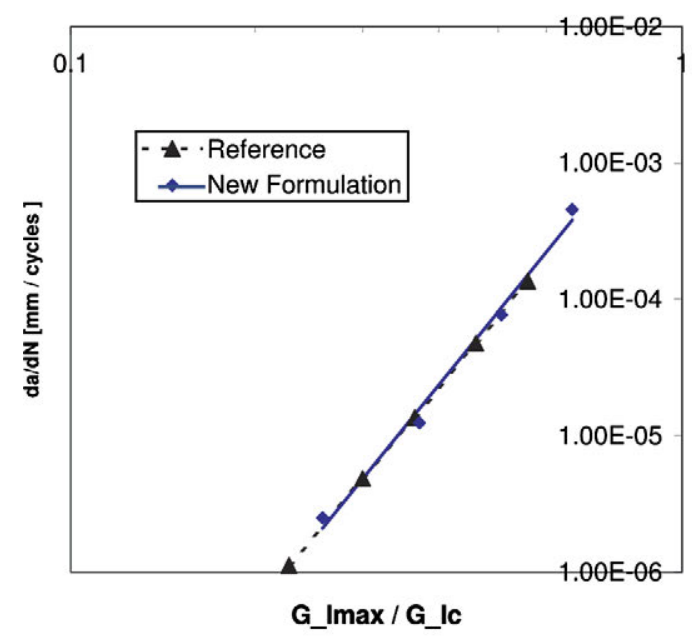

Paris Plot - Mode II

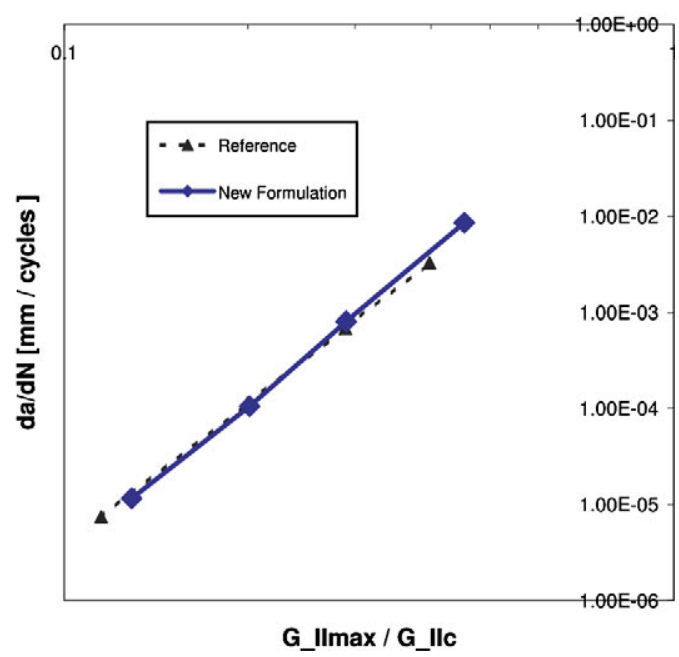

Fig. 13. Comparison between the results published in Ref. [16] and those obtained with the new formulation for mode $\mathrm{I}\left(C_{\text {new }}=3.9 \times 10{ }^{18}, \beta=2\right.$, $\lambda=0.5)$ and mode II $\left(C_{\text {new }}=1.0 \times 10^{19}, \beta=2\right)$.

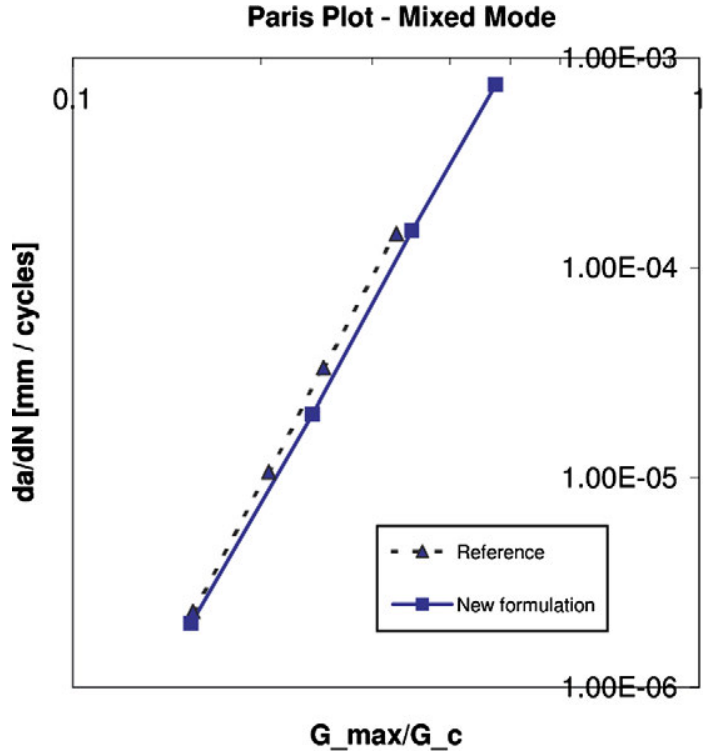

Fig. 14. Comparison between the results published in Ref. [16] and those obtained with the new formulation for mixed mode $\left(C_{\text {new }}=1.0 \times 10^{23}\right.$, $\beta=4.0, \lambda=0.5)$.

$$
\begin{aligned}
H_{i} \equiv & \Delta D_{i}-F_{i}\left(\frac{1}{1+\gamma_{N}}-\frac{1}{1+\gamma_{N+\Delta N}}\right) \\
& -\Delta N C \mathrm{e}^{\lambda D_{i, \mu}} \frac{\left(1+\gamma_{\mu}\right)^{\beta+1}}{1+\beta} .
\end{aligned}
$$

The expression of $\dot{D}_{i, \mathrm{f}}$ in (14) simplifies the linearisation of the stress stain relationship (see Appendix A). Also, it is important to note that the value of the coefficient $C$ must be modified in order to take into account the change of the reference displacement, i.e., the use of $\delta_{0}$ instead of $\delta_{\mathrm{c}}$. In general, the new factor $C$ will be several orders of magnitude smaller than the one used in [16]. For instance, for the realistic values $\frac{\delta_{\mathrm{c}}}{\delta_{0}} \approx 10^{3}$ and $\beta \quad 2$, the new coefficient must be modified as follows:

$C_{\text {new }}=\frac{C}{10^{3(\beta+1)}}=C \times 10^{-9}$.

It is also worth pointing out that the dependence between the crack growth rate $\frac{\mathrm{d} a}{\mathrm{~d} N}$, and $\beta$ and $C$ will be different from the results given in Ref. [16] using the original formulation. Figs. 13 and 14 show that the new formulation proposed in this paper provides results analogous to those generated with the original formulation of Ref. [16]. The results obtained with the alternative formulation are not 'better' or 'worse' than those obtained with the original formulation given in Ref. [16]. The alternative formulation is more consistent from a mathematical point of view and can be more easily applied to hypothetical cases in which an initially simple mode crack propagation becomes mixed or vice-versa.

\section{Conclusions}

The paper studies the source of drawbacks associated with the use of interface elements for fatigue driven delamination problems and, in addition, proposes some improvements with respect to the previous works.

By using some numerical examples, the computational robustness of the formulation described in Ref. [16] has been tested with respect to two significant parameters: the number of cycles per increment $\Delta N$ and the element size $\Delta l$ The numerical results have revealed some limitations on the values of $\Delta l$ and $\Delta N$, which in turn also determine the computational cost of the formulation. Both values are strongly linked, and it has been shown that the use of relatively large interface elements leads to significant oscillations in the crack growth rate. Similar problems have 
been reported in the literature concerning the evolution of the crack for static delamination when using interface elements [8]. This is relevant for the construction of suitable and not unnecessarily expensive numerical models.

The formulation given in Ref. [16] has been extended to include cyclic loads with a non-zero minimum value. This is an important case for real applications and has been incorporated in a straight forward manner. However, an exact relationship between the ratio $\rho=\frac{G_{\min }}{G_{\max }}$ and the coefficient $C$ for fatigue delamination would require more experimental investigations.

Finally, a simplification is proposed for mixed mode fatigue driven delamination growth. The new definition of $\Delta D_{\mathrm{f}}$ avoids the discontinuities in the definition of the stress displacement relationship. The linearised form for the new resulting formulations is given in Appendix A, which require minimal changes with respect to the original work in Ref. [16].

\section{Acknowledgements}

The authors acknowledge the financial support rendered by the European project, COMPASS. The authors also thank FEA Ltd for having provided the code LUSAS.

\section{Appendix A. Linearisation of equations}

From the expressions of $\Delta D_{i}$, in Section 2.2, Eqs. (10), the relation between $\Delta D_{i}$, and $\delta_{i}, i \quad 1,2$, is such that it satisfies

$H_{i}=0, \quad i=1,2$,

where

$$
\begin{aligned}
H_{i} \equiv & \Delta D_{i}-F_{i}\left(\frac{1}{1+\gamma_{N}}-\frac{1}{1+\gamma_{N+\Delta N}}\right) \\
& -\frac{\Delta N C \mathrm{e}^{\lambda D_{i, \mu}}}{1+\beta}\left(\frac{1+\gamma_{\mu}}{1+\gamma_{\mathrm{c}}}\right)^{\beta+1} .
\end{aligned}
$$

Since $\frac{\mathrm{d} H_{i}}{\mathrm{~d} \delta_{j}}=0$, we can write the following relation:

$\frac{\partial D_{i}}{\partial \delta_{j}}=-\left(\frac{\partial H_{i}}{\partial D_{i}}\right)^{-1} \frac{\partial H_{i}}{\partial \delta_{j}}$ that

(Note that $\frac{\partial H_{i}}{\partial D_{j}}=0$ for $i \neq j$.) From (19a) it follows

$$
\begin{aligned}
\frac{\partial H_{i}}{\partial \delta_{j}} & \left.=-\frac{1}{\left(1+\gamma^{*}\right)^{1+\alpha}} F_{i} \frac{\delta_{j}^{\alpha-1}}{\delta_{0 j}}\right)-B \mathrm{e}^{\lambda D_{i, \mu}} \frac{\delta_{j}^{\alpha-1}}{\delta_{0 j}^{\alpha}}, \\
\frac{\partial H_{i}}{\partial D_{i}} & =1-A \mathrm{e}^{\lambda D_{i, \mu}},
\end{aligned}
$$

with

$$
\begin{aligned}
& A=\frac{\Delta N \lambda \mu C}{1+\beta}\left(\frac{1+\gamma_{\mu}}{1+\gamma_{\mathrm{c}}}\right)^{1+\beta}, \\
& B=\frac{\Delta N \mu C}{(1+\gamma)^{\alpha-1}} \frac{\left(1+\gamma_{\mu}\right)^{\beta}}{\left(1+\gamma_{\mathrm{c}}\right)^{1+\beta}} .
\end{aligned}
$$

Inserting Eqs. (21) into Eq. (20) yields

$$
\frac{\partial D_{i}}{\partial \delta_{j}}=\frac{1}{1-A \mathrm{e}^{\lambda D_{i, \mu}}}\left[\left(\frac{1}{\left(1+\gamma^{*}\right)^{1+\alpha}} F_{i} \frac{\delta_{j}^{\alpha-1}}{\delta_{0 j}}\right)+B \mathrm{e}^{\lambda D_{i, \mu}} \frac{\delta_{j}^{\alpha-1}}{\delta_{0 j}^{\alpha}}\right] .
$$

The linearisation of the stress displacement relationship in (2) is therefore expressed as

$\mathrm{d} t_{i}=\left(1-D_{i}\right) K_{i} \mathrm{~d} \delta_{i}-\frac{\partial D_{i}}{\partial \delta_{j}} \mathrm{~d} \delta_{j} K_{i} \delta_{i}=K_{\mathrm{t}}^{i j} \mathrm{~d} \delta_{j}$,

where the component $i j$ of the tangent stiffness matrix $\mathbf{K}_{\mathrm{t}}$ is given by

$$
\begin{aligned}
K_{\mathrm{t}}^{i j}= & \delta_{i}^{j}\left(1-D_{i}\right) K_{i} \\
& -\frac{1}{1-A \mathrm{e}^{\lambda} D_{i, \mu}}\left[\frac{1}{\left(1+\gamma^{*}\right)^{1+\alpha}} F_{i} K_{i} \frac{\delta_{i} \delta_{j}^{\alpha-1}}{\delta_{0 j}}\right) \\
& \left.+B \mathrm{e}^{\lambda D_{i, \mu}} K_{i} \frac{\delta_{i} \delta_{j}^{\alpha-1}}{\delta_{0 j}^{\alpha}}\right],
\end{aligned}
$$

and where $\delta_{i}^{j}=1$ if $i \quad j$ and 0 otherwise.

When considering the alternative coupled formulation in Section 4, the new tangent stiffness matrix is obtained by replacing the term $1+\gamma_{\mathrm{c}}$ with 1 . This is equivalent to using the same expression in (24) but with $A$ and $B$ now given by

$$
\begin{aligned}
& A=\Delta N \lambda \mu C \frac{\left(1+\gamma_{\mu}\right)^{1+\beta}}{1+\beta}, \\
& B=\Delta N \mu C \frac{\left(1+\gamma_{\mu}\right)^{\beta}}{(1+\gamma)^{\alpha-1}} .
\end{aligned}
$$

\section{References}

[1] Rice JR. A path independent integral and the approximate analysis of strain concentrations by notches and cracks. J Appl Mech 1968;35:379 86

[2] Hellen TK. On the method of virtual crack extensions. Int J Num Meth Eng 1975;9:187 207.

[3] Rybicki EF, Kanninen MF. A finite element calculation of stress intensity factors by a modified crack closure integral. Eng Fract Mech 1977;9:931.

[4] Schellekens JCJ, Borst RD. A non linear finite element approach for the analysis of mode I free edge delamination in composites. Int J Sol Struct 1993;30:1239 53 .

[5] Allix O, Ladeveze P, Corigliano A. Damage analysis of interlaminar fracture specimens. Comp Struct 1995;31:66 74 .

[6] Point N, Sacco E. A delamination model for laminated composites. Int J Sol Struct 1996;33:483 509.

[7] Chaboche JL, Girard R, et al. Numerical analysis of composite systems by using interphase/interface models. Comput Mech 1997;33:3 11

[8] Mi Y, Crisfield MA, Davies GAO, Hellweg HB. Progressive delamination using interface elements. J Comput Math 1998;32:1246 72

[9] Alfano G, Crisfield MA. Finite element interface models for delamination analysis of laminated composites: mechanical and computational issues. Int J Num Meth Eng 2001;50:1701 36.

[10] Corigliano A, Allix O. Some aspects of interlaminar degradation in composites. Comput Methods Appl Mech Eng 2000;185:203 24. 
[11] Yang B, Mall S, Ravi Chandar K. A cohesive zone model for fatigue crack growth in quasibrittle materials. Int $\mathbf{J}$ Solids Struct 2001;38:3927 44 .

[12] Suresh S. Fatigue of materials. Cambridge: Cambridge University Press; 1998.

[13] Martin RH, Murri GB. Characterisation of mode I and mode II delamination growth and thresholds in AS4/PEEK composites. Philadelphia: American Society of Testing Materials; 1990.

[14] Dugdale DS. Yielding of steel sheets containing slits. Nonlinear Dynam 1960;8:100 4.

[15] Barenblatt GI. The mathematical theory of equilibrium cracks in brittle fracture. Adv Appl Mech 1962;7:55 129

[16] Robinson P, Galvanetto U, Tumino D, Bellucci G, Violeau D. Numerical simulation of fatigue driven delamination using interface elements. Int J Num Meth Eng 2005;63:1824 48.
[17] Peerlings RHJ, Brekelmans WAM, de Borst R, Geers MGD. Gradient enhanced damage modelling of high cycle fatigue. Int $\mathbf{J}$ Num Meth Eng 2000;49:1547 69.

[18] LUSAS user's manual. Surrey (England): FEA Ltd; 2003.

[19] Roychowdhury S, Dodds RH. A numerical investigation of $3 \mathrm{D}$ small scale yielding fatigue crack growth. Eng Fract Mech 2003;70:2363 83 .

[20] Violeau D, Numerical analysis of delamination fatigue in composites via a cohesive zone model. Department of Mechanical Engineering ENS, Cachan, Master's degree final Report; 2001.

[21] Asp LE, Sjgren A, Greenhalgh ES. Delamination growth and thresholds in a Carbon/Epoxy composite under fatigue loading. J Comp Technol Res 2001;23:55 68.

[22] Vasudevan AK, Sadananda K, Glinka G. Critical parameters for fatigue damage. Int J Fatigue 2001;23:S39 53. 